\title{
The Delayed Quantum Eraser Experiment Explained Classically
}

\author{
Declan Traill \\ Independent Researcher, Melbourne, Australia \\ Email: declan@netspace.net.au
}

How to cite this paper: Traill, D. (2021) The Delayed Quantum Eraser Experiment Explained Classically. Journal of Modern Physics, 12, 1183-1189.

https://doi.org/10.4236/jmp.2021.129072

Received: June 3, 2021

Accepted: July 2, 2021

Published: July 5, 2021

Copyright $\odot 2021$ by author(s) and Scientific Research Publishing Inc. This work is licensed under the Creative Commons Attribution International License (CC BY 4.0).

http://creativecommons.org/licenses/by/4.0/

\begin{abstract}
This paper discusses the well-known delayed choice Quantum Eraser experiment performed by Kim et al. in 2000 and analyzes it from a Classical Physics perspective. I have included a diagram of the setup used in the experiment. I show that the result of the experiment can be explained by Classical Physics and does not require "Spooky action at a distance" due to entangled particles, as Einstein famously once put it, nor events modifying the past due to the delayed choice aspect of the experiment.
\end{abstract}

\section{Keywords}

Delayed, Choice, Quantum, Eraser, Photon, Laser, EPR, Entangled, Classical, Physics, Polarizing, Beam, Splitter, Coincidence, SPDC

\section{Introduction}

This paper is the second I have written on analyses of key experiments in Quantum Optics that claim to employ entangled photons in the experimental setup, resulting in correlations in photon detections which are claimed to demonstrate the Quantum nature of the behaviour of the light in the experiments. It is claimed by these experimental results that no Local, Real (Classical Physics) explanation for the results is possible, and the design of these experimental setups is done in such a way to supposedly close any loopholes that would allow for a Classical explanation.

In previous research I have conducted [1], I examined modern versions of the original Einstein-Podolsky-Rosen (EPR) thought experiment of Quantum Mechanics. The EPR experiment is thought to demonstrate that photons of light can become entangled when they are created by a process such as Spontaneous Parametric Down Conversion (SPDC) and then can travel on to different, spa- 
tially separated, regions within the laboratory and then, upon measurement, display correlations in their respective polarizations despite their separation being such that a light signal cannot communicate between these two locations within the duration of each measurement.

In my examination and computer modelling of this experiment, I found that the correlation curve obtained in the experiment can be completely explained by a detection bias at certain detector polarization angles such that as the detector is rotated through 180 degrees, a cosine shaped correlation curve is obtained, rather than the linear curve which was expected from a Classical model. The Quantum Mechanical prediction, based on there being entanglement between the photons, is also a cosine curve thus giving confidence in the Quantum Mechanical interpretation. The experiment I examined used a Steering Inequality to assess the level of correlation between the photons in the experiment. Such a Steering Inequality is supposed to be a better method of assessing the correlation than the Bell or $\mathrm{CHSH}$ inequalities that are usually used and is claimed to close the detection-loophole (the detection bias that I modelled in my analysis). However, in my Classical model, I found not only did the model result in the same cosine curve that Quantum Mechanics predicts, but the Steering Inequality is comprehensively violated too. My model found a Steering Inequality of 1.622, yet a Classical model in only supposed to be able to give a Steering Inequality of 1 or less.

The experiment performed by Kim et al. [2] is a variation on this EPR type of experiment. Their experiment is called a Delayed Choice Quantum Eraser experiment and was devised to deliberately make one of the photon path lengths longer than the other such that one photon's polarization can be known before the other is measured. The rationale is that it is thought that by measuring the 2nd photon, the "which path" information of the photon becomes known and the wave-function of the entangled photon pair collapses, so by delaying its measurement until after the 1st photon has either interfered (or not) it should not be able to influence the 1st photon's measurement without signaling backward in time. Therefore, so the reasoning goes, if there is a correlation between the path taken by the 2nd photon and the pattern of the 1st photon (interfering or otherwise) then that indicates Quantum weirdness that cannot be explained with a Real, Local Physical model. Having successfully modelled the EPR experiment as a completely Classical process, I had my doubts as to whether there was really any Quantum entanglement required, or indeed happening at all; so, I thought it might be useful to turn my attention to the Delayed Choice Quantum Eraser experiment too and see if a rational Classical explanation might also exist.

\section{Aim}

The aim of this paper is to demonstrate that the usual assumptions made in the analysis of this experiment are incorrect and that a simple, real and local explanation exists that can fully explain the results of the experiment without the need to resort to faster-than-light nor backwards-in-time communication. 


\section{Method}

The main features of the Kim et al. [2] setup shown in Figure 1 are:

- The pulsed input laser beam striking a double-slit barrier.

- The photon output from the double-slit enters a BBO crystal where SPDC occurs.

- The "entangled" pairs of photons from the SPDC process are split down two paths by a Glan-Thompson beam splitter.

- The two signal photons (one from each pair) are converged with a lens onto detector D0 (which can be moved in the $\mathrm{x}$-axis direction to observe the interference fringes)

- The idler photons (one from each pair) are further separated by a prism.

- Each idler photon passes through a polarizing beam splitter BSa or BSb.

- The idler photons then get recorded at either D3 or D4, or pass through a further beam splitter BSc and then off mirrors $\mathbf{M a}$ or $\mathbf{M b}$ to detectors D1 or D2.

- Detection results are recorded and correlated by a coincidence counter.

The main feature of the experimental setup is the initial photon from the pulsed laser passing through a double-slit, thus presenting an unknown path aspect to the situation, followed by the generation of entangled photons-one pair from each photon path through the double-slit. Then one photon from each pair (the signal photons) passes down a short path and are brought together and allowed to interfere, whilst the other photon from each pair (the idler photons) travels a longer distance and passes through several beam splitters before reaching 4 possible detectors ( 3 of the 4 are possible detectors for each idler photon). Due to the path length difference, the result of the signal photons' interference is

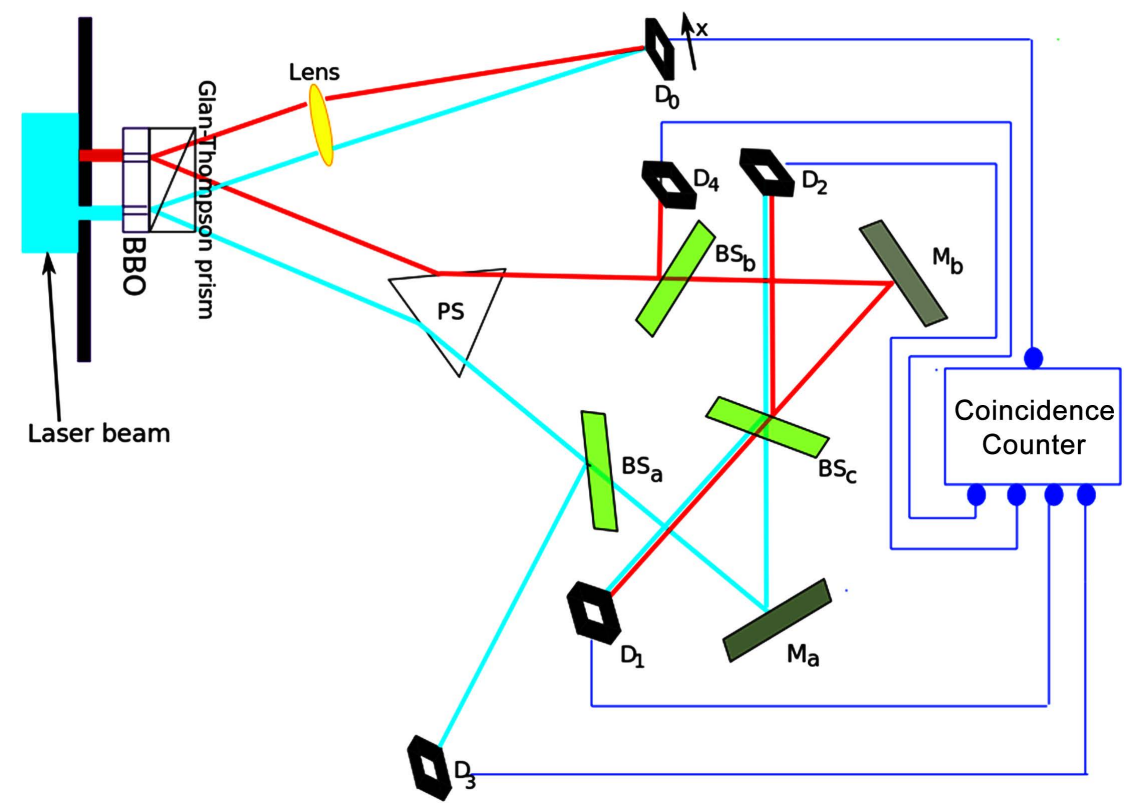

Figure 1. The experimental setup used by Kim et al. [2]. Attribution for this image is given in Ref. [3]. 
known before the path taken result (indicated by the detections of the idler photons) is known. The reasoning is that if knowing the path of the idler photons can collapse the Quantum Mechanical wave-function and affect the interference result of the signal photons, then by delaying the knowledge of the path taken until after the interference result is known any strange correlation in the results of all the photons in the experiment might be prevented. The Figures in the Kim et al. paper [2] do display a correlation that is thought to indicate entanglement between photons.

\section{Analysis of Results}

In order to analyze this experiment from a new, objective perspective I tried to imagine exactly what the light waves were doing at every point through the experimental apparatus. I aimed to keep Classical Physics front of mind and avoid having to resort to any non-real, non-local explanation for the observed results. One key feature of the experiment is that it used brief pulses of laser light such that single photons were traversing the experimental setup during the data collection. This is one of the features that make the results so confusing to understand, especially if one thinks of photons as discrete point particles.

The first thing one must realize in order to make sense of the experiment is that photons are not discrete particles but are actually continuous Electromagnetic waves. Sure, atoms emit and absorb Electromagnetic energy in fixed quantum amounts due to the changes in electron orbital energy levels, which gives rise to the wave packets of Electromagnetic energy that we refer to as Photons, but there is nothing binding this wave energy into discrete particles. Indeed, the wave packets that are referred to as photons may be meters long (depending on the frequency of the light) and are not point particles as is often assumed. Thus, when "photons" of light pass through a double-slit or a beam splitter the wave energy is free to be split up into different sub-quantum amounts down different paths.

We can see that this in fact must be the case when we observe single photons passing through Mach-Zehnder Interferometers [4] [5]. When single photons of light pass through such interferometers, interference patterns are still observed in the output when one of the path lengths in the Interferometer is altered to make it shorter or longer that the other path. This could only happen if Electromagnetic energy was passing down both paths simultaneously.

Thus, in the case of the experiment conducted by Kim et al. [2] (also see Figure 1 above), the light photons will arrive at the double slit at the start of the experiment and, depending on the exact position and polarization of the photon, will either pass entirely (or almost entirely) through one of the two slits, or will diffract through both slits (with varying proportions of energy passing through each). When the latter occurs, sub-quantum amounts of Electromagnetic energy emerge from both slits A and B and proceed on to the BBO crystal. The process of SPDC which occurs in this crystal to convert $351.1 \mathrm{~nm}$ light into two beams of $702.2 \mathrm{~nm}$ light works based on the conservation of energy and momentum [6], 
so the same laws will apply to the sub-quantum light waves that emerge from the double-slit and will result in sub-quantum $702.2 \mathrm{~nm}$ photons being generated in the BBO crystal.

After the BBO crystal, the sub-quantum $702.2 \mathrm{~nm}$ photons will continue through the apparatus to the beam splitters. The beam splitters are polarizing beam splitters that separate out orthogonal polarization components down their two output channels (causing either transmission or reflection). So, the photons will either transmit or reflect at these beam splitters depending on their polarization axes relative to the polarization axes of the beam splitters. It should be noted that the detection of light quanta by the detectors is a probability driven event that is based on the probability amplitude of the light signal. Thus, even though a sub-quantum amount of light is received at a detector, the probability of a detection event occurring at the detector increases with the amplitude of the received light signal. Thus, even sub-quantum amounts of light can cause detectors to trigger and generate "click" events.

There seems to be the mistaken assumption in the usual analysis of this experiment that 50:50 beam-splitters will separate each photon down each output channel with a 0.5 probability down either channel. This is NOT correct (unless non-polarizing beam splitters were used in the experiment). With unpolarized light beams (consisting of many photons with random polarization directions) there will be a 50:50 split in beam intensity down each channel, but for individual photons with specific polarizations the output channel is determined by the relative orientations of the polarization axes of the light and the beam-splitter. The choice of which of the two paths taken is not a 50:50 chance, but would range between 100:0 and 0:100 depending on the relative polarization of the photons with respect to the optical axes of the polarizing beam splitters.

As the SPDC process [6] used in the experiment produces photons with orthogonal optical axes (a type II SPDC process) the detections observed in the experiment are simply due to the two photon pairs produced having the same or different polarizations down the paths to the detectors.

So, if two orthogonal signal photons are produced down the path to detector Do then there will be no interference at that detector (as orthogonal light beams of photons do not interfere). The orientation of the optical axes of the other two photons, the 2 nd photons (known as the idler photons) from the two pairs produced, is such that one photon will reflect and the other will transmit at the beam splitters BSa and BSb as these beam splitters are polarizing and will separate out different polarizations down each of their output paths.

Hence the observed detection curve $\mathrm{R}_{03}$ or $\mathrm{R}_{04}$ in Figure 2, showing no interference pattern, is obtained, (in the original Kim et al. paper [2] it is Figure 5 on page 4$)$.

Then, if two photons (one from each pair produced), each having the same optical axis orientation, are sent down the path to detector D0, they will interfere at that detector. Assuming the optical axis of the other two photons (orthogonal to the first two) is aligned with the optical axis for transmission in BSa and BSb, 


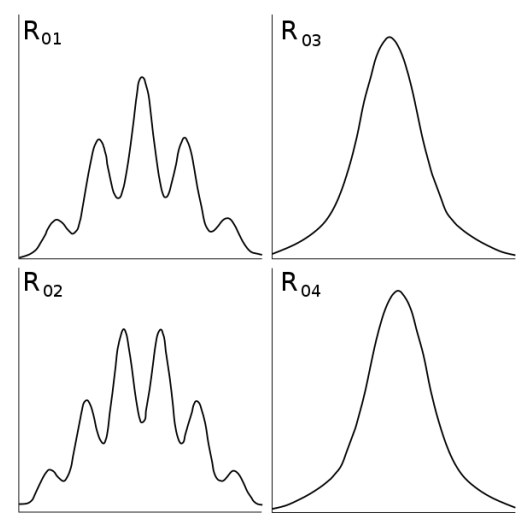

Figure 2. The experimental correlation results obtained by Kim et al. [2]. Attribution for this image is given in Ref [7].

they will always transmit through the beam splitters $\mathbf{B S a}$ and $\mathbf{B S b}$ and go on to be recorded at detectors D1 or D2.

Thus, the detection results $R_{01}$ and $R_{02}$ in Figure 2, showing a correlation curve that has an interference pattern, are observed (as shown in Figure 3 \& Figure 4 of the original Kim et al. paper [2]).

The raised center in the observed interference pattern of the joint-detection graphs of $\mathrm{R}_{01}$ and $\mathrm{R}_{02}$ in Figure 2 is due to some of the "click" events at detectors $\mathrm{D} 1$ and $\mathrm{D} 2$ being due to the single idler photon from an orthogonal pair being transmitted at BSa or BSb (when the other two signal photons going to detector D0 are also orthogonal to one another, so don't generate any interference pattern at detector D0). The result of such detections is evident in the two figures graphs $R_{01}$ and $R_{02}$ in Figure 2, as the interference fringe peaks vary in height (and from a different base level) following the shape of the curve seen in graph $\mathrm{R}_{03}$ in Figure 2. There would also be some contributions to the non-interfering photons detections (giving rise to this non-interfering component of the detection curve) from single photons that pass entirely through one of the slits at the start of the experiment and then go on to detectors D1, D2, D3 or D4.

\section{Conclusion}

Therefore, we can see that once the nature of the SPDC process (generating orthogonally polarized photons) and the nature of how polarizing beam splitters work are taken into consideration, the results of the experiment are consistent with a Classical Physics (Local and Real) interpretation and there is no mystery requiring the notion of entangled pairs, nor faster than light communication. There is also a classical explanation for the original EPR experiment [1] that can explain the correlation in experimental results without requiring any notion of "entanglement" of the photons (or other particles such as electrons [8]).

\section{Conflicts of Interest}

The author declares no conflicts of interest regarding the publication of this paper. 


\section{References}

[1] Traill, D.A. (2018) A Fundamental Misunderstanding (fqXi). https://www.researchgate.net/publication/327260788 A Fundamental_Misundersta nding

[2] Kim, Y.-H., Yu, R., Kulik, S.P., Shih, Y.H. and Scully, M. (2000) Physical Review Letters, 84, 1-5. https://doi.org/10.1103/PhysRevLett.84.1

[3] Noestmm, PNGHUT.

https://pnghut.com/png/CW8jEALu4U/quantum-eraser-experiment-double-slit-del ayed-choice-wave-particle-duality-wheelers-wave-transparent-png

[4] ScienceDirect, "Mach-Zehnder Interferometers" (2020) Elsevier B.V. https://www.sciencedirect.com/topics/physics-and-astronomy/mach-zehnder-interf erometers

[5] Khokhlov, D.L. (2019) Quantum Information Review, 7, 7-10. http://www.naturalspublishing.com/files/published/f721wno893296q.pdf

[6] Wikipedia, "Spontaneous Parametric Down-Conversion". https://en.wikipedia.org/wiki/Spontaneous parametric down-conversion

[7] Delayed Choice Quantum Eraser Graphs.svg, 2014, Wikimedia, Stigmatella aurantiaca at English Wikipedia, CC BY-SA 3.0.

https://creativecommons.org/licenses/by-sa/3.0 https://commons.wikimedia.org/wiki/File:Delayed Choice Quantum_Eraser_Grap hs.svg

[8] Jackson, P.A. (2021) The Measurement Problem, an Ontological Solution. https://www.researchgate.net/publication/352056822 The Measurement Problem an Ontological Solution 